\title{
Articulo
}

\section{Las Tecnologías de la Información y la Comunicación y su contribución en la construcción de las Ciudades Digitales}

\author{
*Autor: M.Sc. Jairo Auqusto Ortegón Bolívar* \\ Universidad Autónoma de Colombia \\ Colombia-jortegonb@yahoo.com
}

\author{
"La ciudad no es una jungla de hormigón, es un zoo humano." \\ **Desmond Morris, 1960
}

Lugar: Bogotá DC, ciudad capital de la Republica de Colombia

Fecha de presentación: Marzo 20 de 2012

*Notas sobre el autor:

jortegonb@yahoo.com

Jairo Auqusto Ortegón Bolívar: Ciudadano Bogotano de profesión Ingeniero Electrónico, Especialista en Administración, Magíster el Telecomunicaciones e Informática -Teleinformática. Su trayectoria profesional se ha desarrollado tanto con la empresa privada como con el estado. Entre otras entidades ex-funcionario del Ministerio de Comunicaciones y de la Empresa Nacional de Telecomunicaciones TELECOM. Docente Investigador de la Universidad Autónoma de Colombia y otras universidades en las Cátedras de Modulación de señales, Sistemas de Telecomunicaciones, Antenas y Medios de Propagación de señales e Ingeniería de las Telecomunicaciones, Legislación en Telecomunicaciones, Televisión digital. Actualmente cursa el Programa de Doctorado sobre la Sociedad de la Información y el Conocimiento con énfasis en Tecnologías de la Información y la Comunicación y Gobierno Electrónico en la Universidad Oberta de Cataluña UOC.

** Desmond Morris: zoólogo y etólogo inglés. Nació en 1928 en Purton, Wiltshire, en la campiña inglesa. Sus estudios se centran en la conducta animal, y por ende, en la conducta humana, explicados desde un punto de vista estrictamente zoológico. Su libro más conocido, The Naked Ape (El mono desnudo), publicado en 1967, es una realista y objetiva mirada a la especie humana. El zoo humano, continuación de El mono desnudo, examina el comportamiento humano en las ciudades, también desde un punto de vista etológico. 


\section{RESUMEN}

El presente artículo pretende connotar el hecho de cómo las Telecomunicaciones y sus diversas aplicaciones, han contribuido al origen, implementación, desarrollo y sostenibilidad de lo que hoy denominamos Ciudades Digitales. La Teleinformática desde sus inicios y su posterior desarrollo se ha convertido en el soporte de la aplicación de las Nuevas Tecnologías de la Información, más conocidas como Tecnologías de la Información y la Comunicación TIC, las cuales han transformado la antigua sociedad industrial, generando dentro de la sociedad humana nuevas relaciones económicas, políticas y sociales centradas en el intercambio de flujos de información, que han derivado en la configuración de un nuevo modelo social conocido con el nombre de Sociedad Postindustrial o Sociedad del Conocimiento. En consecuencia dentro de un ambiente global este fenómeno tecnológico de las TIC ha transformado las relaciones económicas, sociales e incluso las relaciones de poder entre los países.

Se revisara históricamente como las TIC ha venido siendo soporte para constituir la nueva estructura física de la que se han revestido nuestras ciudades originales en una forma similar a las estructuras compuestas por subsistemas que poseen los organismos vivos, valga decir: esqueletos estructurales, varias capas de piel protectora y sistemas nerviosos artificiales. Se muestra entonces que para crear la nueva inteligencia de las ciudades y esto facilite la capacidad de gestión, hay que combinar el software con las redes de telecomunicaciones, los sensores y los identificadores dentro de un ambiente digital, proveyendo entonces una inteligencia integrada a estas ciudades de una forma ubicua que les permitan responder de manera inteligente a las demandas de los diferentes entornos dentro de los cuales se encuentran integradas.

\section{Palabras clave}

Telecomunicaciones, Informática, Tecnologías de la Información y la Comunicación, Conectividad, Ciudades Inteligentes, Conexiones sociales.

\section{Tema}

Inteligencia, Conectividad y Convergencia

\section{Abstract}

This article aims to connote the fact of how Telecommunications and its various applications, have contributed to the origin, implementation, development and sustainability of what is now called Digital Cities. The Tele since its inception and its subsequent development has become the support of the implementation of New Information Technologies, better known as Information Technology and Communication ICT, which have transformed the old industrial society, resulting inhuman society new economic, political and social focus on the exchange of information flows, which have resulted in setting up a new social model known Postindustrial Society or Knowledge Society. Thus within a global environment this technological phenomenon of ICT has transformed the economic, social and even the power relations between countries.

Revise ICT historically has been being supported to form the new physical structure of which have been coated our cities original in a manner similar to the structures composed of subsystems which have living organisms, it is worth saying structural 
skeletons, several layers of protective skin and artificial nervous systems. Is then shown that the new intelligence to create cities and this facilitates the management capacity, we must combine software and telecommunications networks, sensors and identifiers within a digital environment, providing then a built-in intelligence to these cities a ubiquitously to enable them to respond intelligently to the demands of different environments within which they are integrated.

\section{Key words}

Telecommunications, Computer Science, Intelligent Technologies of the Information and the Communication, Connectivity, Cities, Social Connections.

\section{Subject}

Intelligence, Connectivity and Convergence

\section{Introducción}

Para ubicar el tema de las Ciudades Inteligentes y su relación con las $\mathrm{TIC}^{1}$ tanto las previas como las actuales en proyección, empecemos por tomar la definición contenida en el Manual para el desarrollo de Ciudades Digitales en Iberoamérica, elaborado por AHCIET (Asociación Hispanoamericana de Centros de Investigación y Empresas de Telecomunicaciones) el cual concibe a una Ciudad Digital como "aquella en la que, utilizando los recursos que brindan la infraestructura de telecomunicaciones y de informática existentes, entre ellas la denominada Internet, brinda a sus habitantes un conjunto de servicios digitales a fin de mejorar el nivel de desarrollo humano, económico y cultural de esa comunidad, tanto a nivel individual como colectivo". Por consiguiente para tener claridad de como las TIC han podido y continuaran aportando en la construcción de estas Ciudades Inteligentes debemos ver estas construcciones digitales en proyección, y para ello es necesario mirar el extenso proceso evolutivo que han sufrido las antiguas ciudades. La arquitectura física de las antiguas ciudades previas incluso a la revolución industrial, consistían esencialmente en una estructura compuesta por calles, columnas, vigas, paredes, y tejados. Su función primaria consistía en facilitar hospicio, protección, e intensificar el uso de la tierra (Caragliu, 2009).

Sus habitantes realizaban transacciones sociales y económicas face to face y suministraban la inteligencia necesaria para hacer operar la ciudad como un sistema (Vélez, 2000). Con la industrialización, las ciudades lograron adquirir fisiologías artificiales cada vez más extensas (Grafica No 1). Empezaron a ser más estructuradas puesto que ya contaron formalmente con las redes de suministro de agua y de evacuación de residuos líquidos, sólidos y gases, redes de suministro de energía eléctrica, redes de transporte y redes de calefacción y aire acondicionado en los edificios. Habitar una ciudad significaba estar continuamente conectado a estas redes y depender de ellas para sobrevivir. Entretanto, avanzado el siglo diez y nueve, las ciudades comenzaron a añadir sistemas nerviosos artificiales a sus ya existentes estructuras arquitectónicas y de redes de suministro, procesamiento, evacuación y fueron construyendo e

\footnotetext{
${ }^{1}$ Cuando nos referimos a las tecnologías de información TI previas a las TIC, se trata de las ciencias de la informática (computación, redes alambricas e inalámbricas, software), las audiovisuales (TV, radio, cine, videos) e incluso el libro, la prensa escrita, el correo postal entre otros como formas de difusión de información convencionales. En cuanto a las TIC actuales, se definen como "el conjunto de actividades de investigación, desarrollo, fabricación, integración, instalación, comercialización y mantenimiento de componentes, subconjuntos, productos y sistemas físicos y lógicos, fundamentados en la tecnología electrónica, así como la explotación de servicios basados en dichas tecnologías, la producción y difusión de contenidos soportados electrónicamente y las aplicaciones de Internet" (Banegas, 2000).
} 


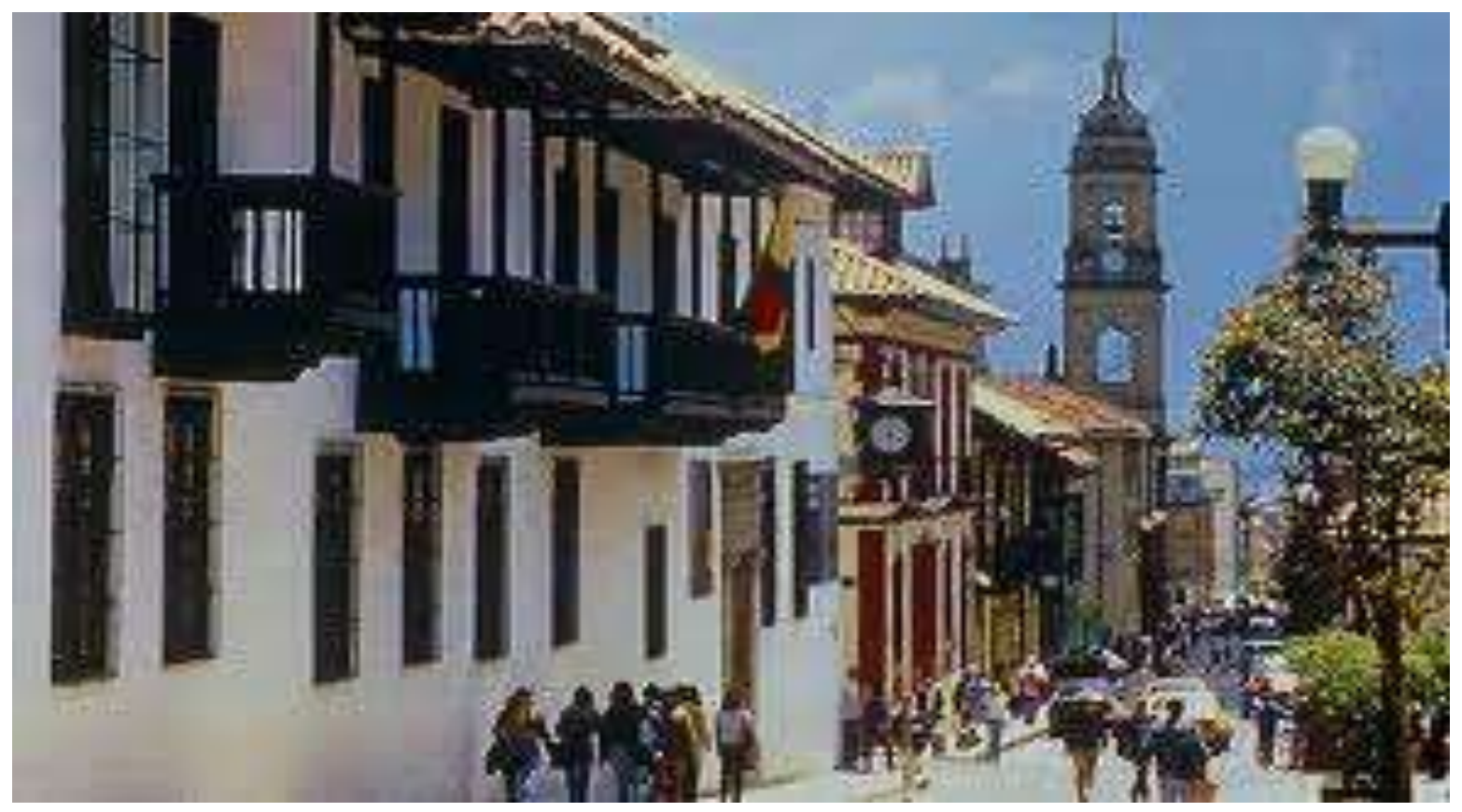

(Grafica No 1). Bogotá - Antigua. La Candelaria. Tomada de: transportationceranayom.qapacity.com

instalando sistemas de telecomunicaciones basados en técnicas análogas tales como el telégrafo, el teléfono, la radio, la televisión apoyadas o no por enlaces o segmentos satelitales y luego se acelero este proceso con la aparición y puesta en práctica de las técnicas de telecomunicaciones digitales hasta lograr tener una ciudad interconectada (Grafica No 1).

\subsection{Aporte de las Telecomunicaciones en las Ciudades Digitales}

Acerca de esta ola digitalizadora, en primera instancia emergió el desarrollo de la teoría de la información digital de Claude Shannon, haciéndose evidente su aplicación entre otras tantas técnicas como la Modulación por Codificación de Pulsos PCM, seguida en la década de 1960 por la invención de la conmutación de paquetes, combinada con la rápida expansión de las técnicas de Múltiplexación Digital de canales de comunicación, ISDN (red digital de servicios integrados) ya sea utilizando los medios radiados (espectro electromagnético) o utilizando los medios guiados ( cable coaxial, fibra óptica entre otros), la ARPANET, INTERNET Y LA WORLD WIDE WEB. Esto puso en su sitio a los circuitos nerviosos en la cantidad necesaria tanto en edificios (cableado estructurado y dispositivos de comunicación) como en el ámbito ciudadano local, luego nacional (redes de fibra óptica) y finalmente global ( cables submarino y enlaces satelitales), facilitando entonces que por esos nervios artificiales de la ciudad fluyeran mayores cantidades de información a través de circuitos conmutados, circuitos de paquetes, circuitos virtuales, circuitos dedicados y luego a través de redes plesiocrona (PDH), redes síncronas(SDH O SONET), modos de transmisión asíncrona (ATM) que conforman lo que hoy llamamos Redes de Alta Velocidad para continuar con las denominadas Redes de Nueva Generación (RNG) basadas en las familias de los protocolos IP y procesamientos con circuitos virtuales MPLS(multi protocol level switchting). Sin embargo paralelamente a lo anterior, se lograron desarrollos tecnológicos que llegan a su máxima expresión en cuanto a conectividad y convergencia hacia finales los noventa del siglo veinte con la proliferación de las diferentes generaciones de sistemas de telecomunicaciones que utilizan técnicas inalámbricas como las 
redes de telefonía móvil (GSM, CDMA, PCS, UMTS, LTE) inicialmente las terrestres y luego las satelitales, radiolocalizadores (GPS), redes inalámbricas (LMDS, BLUETOOTH, WI-FI, WIMAX), los identificadores RFID (identificadores digitales de radio frecuencia). Si recordamos a Marshall McLuhan pionero teórico de los media, le damos la razón cuando como con una mirada prospectiva denominaba a estas redes de telecomunicaciones, como extensiones de los sistemas nerviosos humanos.

No se puede olvidar que durante las década de 1970 al 1980, llegaron los efectos cada vez más profundos de la revolución de la electrónica con la utilización de la ola semiconductora. Consecuencia de la misma, los computadores o MAIN FRAME, que hasta entonces habían sido grandes, delicados, caros y limitados a unos pocos sitios especializados y privilegiados. Lugares que por cierto parecían grandes peceras y era donde invitábamos con vanidad a nuestros amigos, pasaron a ser mucho más pequeños, mucho menos caros y más robustos.

Hacia la mitad de la década de 1980, este desarrollo hizo que los computadores de escritorio hicieran parte normalmente de la vida diaria, y éstos, pronto se conectaron a las redes digitales, cada vez más extensas (las conocidas redes PAN, LAN, MAN, WAN). Y luego con la miniaturización y mejoras posteriores en elevaron el rendimiento de los dispositivos semiconductores. Caso especial de desarrollos con semiconductores tenemos la aparición y desarrollo de los MICROPROCESADORES, y luego los MICROCONTROLADORES, mejorados en grado mayor con base la inclusión de los FPGA y FPAA, lo que dio paso a varios dispositivos modernos como los computadores portátiles, los teléfonos móviles inteligentes o de última generación, las CÁMARAS DIGITALES, los BLACKBERRY, los IPOD, las TABLETS. Estos desarrollos trajeron como consecuencia que la inteligencia digital ya no estuviese tan concentrada, sino que ahora estaba presente de forma transversal, vertical en todos los entornos urbanos de nuestra vida diaria, desde luego en los sitios donde el nivel económico, le permitiera a nosotros los mortales tener acceso a los mismos (Grafica No 2).

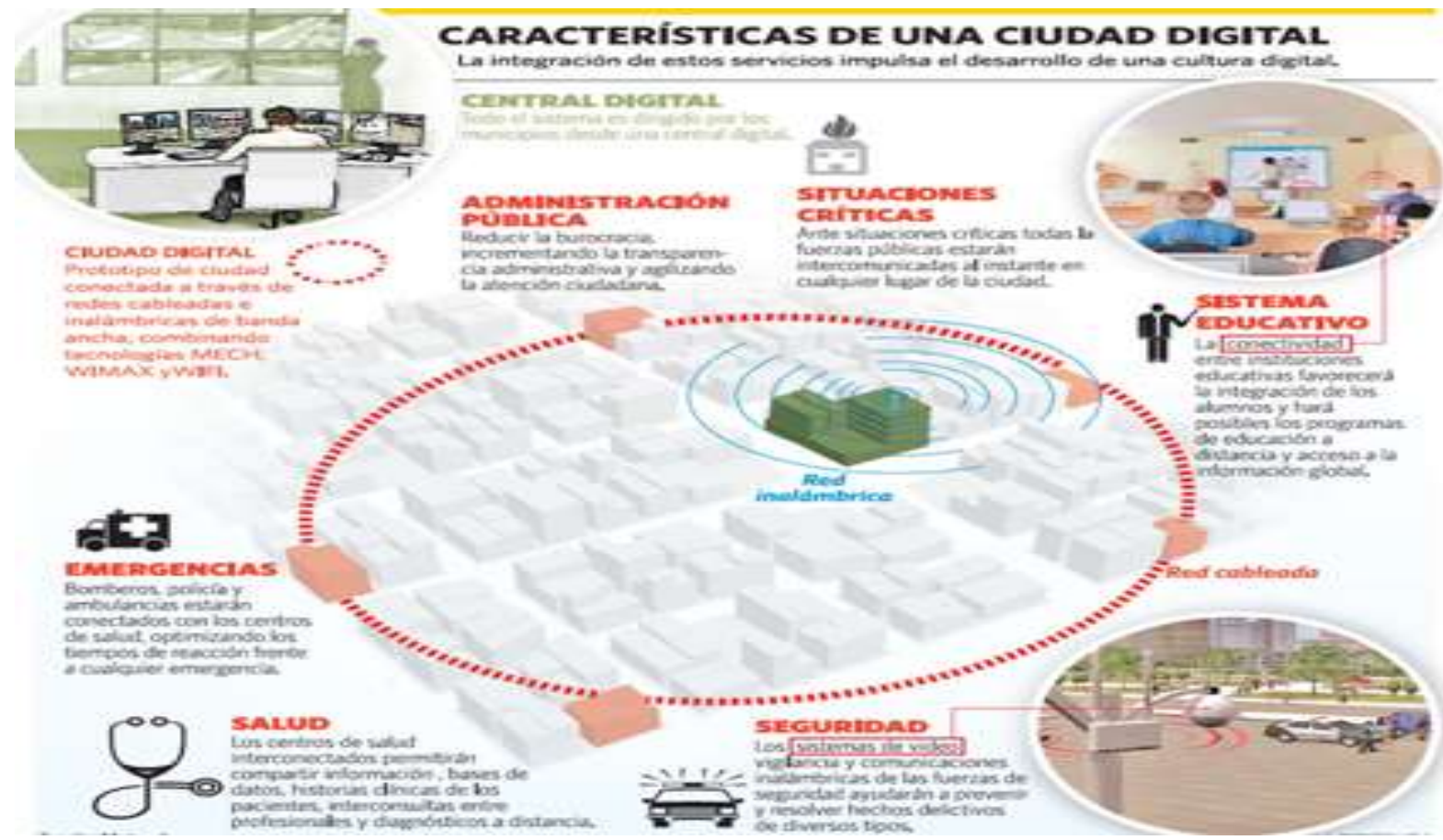

Grafica No 2. Características de una Ciudad Digital. Tomada de: lavidaeninternet.net 
Como hemos revisado, los elementos de inteligencia urbana artificial no aparecieron de una vez por todas, ha sido un complejo proceso de emergencia e integración tecnológica en sistemas cada vez de mayor jerarquía pudiendo hacerse un símil con la evolución biológica, las estructuras existentes y las mutaciones inesperadas o provocadas que se utilizan para nuevos propósitos dentro de organizaciones funcionales emergentes.

En el libro de William J. Mitchell, titulado "The Cyborg Self and the Networked City" denota como estas extensiones artificiales de los sistemas nerviosos humanos permitían a las ciudades experimentar cambios en sus entornos internos, externos y responder, como organismos, de un modo coordinado inteligentemente. De esta forma las ciudades poseían todos los subsistemas cruciales de los organismos vivos: esqueletos estructurales; redes de entrada, procesamiento y eliminación de aire, agua, energía y otros elementos esenciales, y múltiples capas de piel protectora (Mitchell, 2007).

\subsection{Aportes de la Informática en las Ciudades Digitales}

En cuanto a la Informática vemos que la Ingeniería de Software a partir de los setenta del siglo veinte su contribución ha sido decisiva ya sea con desarrollos propietarios o de software libre en la construcción de esas, las Ciudades Digitales. La contribución de la Informática es evidente, múltiple e infinita en todos los desarrollos y aplicaciones del mundo WEB (CONSTRUCCIÓN DE PORTALES, TRANSFERENCIA DE ARCHIVOS, ACCESOS A BASES DE DATOS, ACCESOS A OTRAS REDES, CORREO ELECTRÓNICO, MENSAJERÍA, CHAT, WIKIS, BLOG, SEGURIDAD DE LA INFORMACIÓN, ETC.). sin embargo al final de la década de 1990, se pensó que con la explosión de la burbuja puntocom que la era digital se trataba sólo de la conectividad por Internet, de los computadores personales y los sitios WEB. Se suponía entonces que con estos desarrollos, la supuesta muerte de la distancia, la desmaterialización de casi todo había llegado, pero la emergencia de nuevas oportunidades de negocio motivaron otros desarrollos tecnológicos, aspectos que se manifestaron en acciones como el COMERCIO ELECTRÓNICO a través de desarrollos como los EBAY, TRANSFERENCIAS ELECTRÓNICAS, compras en línea con tarjeta utilizando protocolos SET (Transacciones Electrónicas Seguras) u otras aplicaciones. Pero mientras tanto y en forma paralela, una tercera ola de innovación tecnológica se desarrollaba, la de los SENSORES E IDENTIFICADORES DIGITALES. Los cuales hacían notar su presencia en asocio con otros dispositivos tales como las minúsculas cámaras digitales, los micrófonos, lo que permitió dotar a Internet de ojos y oídos complementando cada vez más ese cuerpo digital de las ciudades. Los GPS y otras tecnologías de localización permitieron a aparatos como los automóviles y los teléfonos móviles saber en todo momento cual era su ubicación en tiempo real (Grafica No 3).

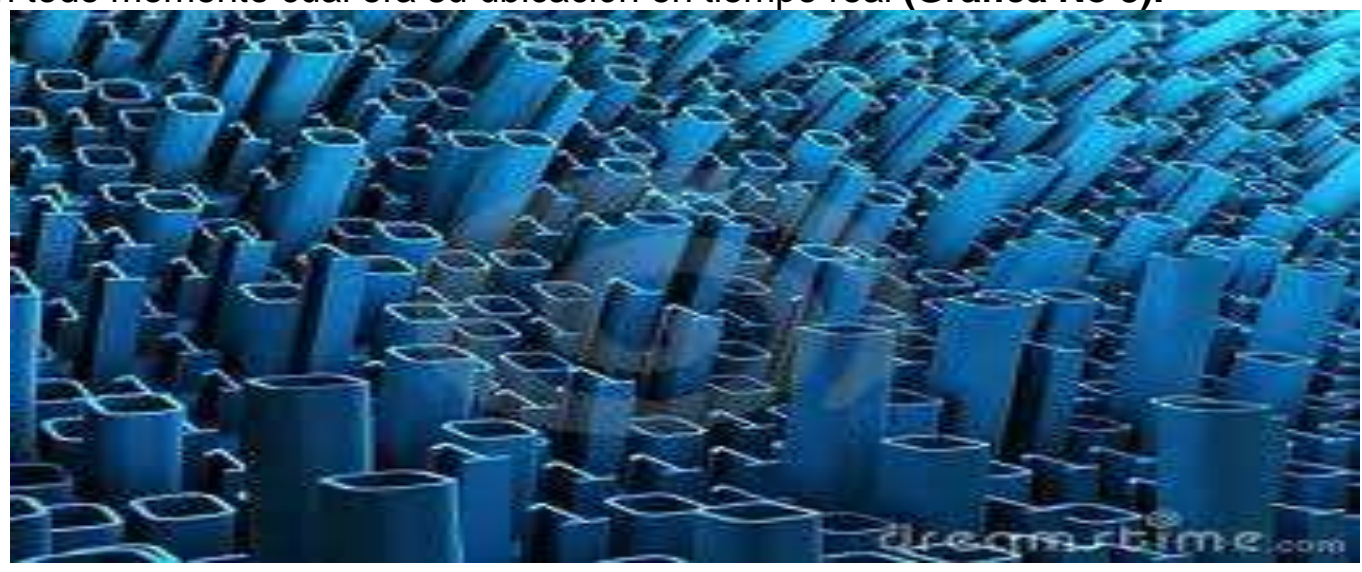

Grafica No 3. Ciudad Digital Binaria, Fondo Abstracto de la Tecnología 3d. Tomado de: es.dreamstime.com 
Los identificadores RFID integrados en productos y embalajes empezaron a revolucionar la logística y la venta al detal, logrando en conjunto obtener la capacidad de percibir y responder rápidamente a condiciones y acontecimientos en el mundo físico. Es así, como estas aplicaciones basadas en hardware electrónico tuvieron como efecto el de tejer una compacta telaraña de conexiones entre el ahora global sistema nervioso artificial y el mundo físico.

\subsection{Aportes de las TIC en las Ciudades Digitales}

Vemos entonces en forma palpable como la contribución de la Teleinformática a través de desarrollos de Ingeniería de Software a gran escala desplegados a su vez a través de medios de telecomunicaciones unen todas estas piezas para funcionar como sistemas coordinados inteligentemente y distribuidos geográficamente. Los cuales son hoy en día cruciales e ineludibles en la vida urbana diaria. Son muchos más los innumerables ejemplos sobre la aplicación de la Teleinformática que muestran la consolidación de jerarquías cognitivas y de gestión similares a las manifestadas en las operaciones de la mente humana que se pueden mostrar, y que está presente su contribución en el ámbito global. Basta ver, el inmenso y sofisticadísimo aparato de software de los exploradores de Mozilla Firefox, o de Internet Explorer o la versatilidad de Google, Altavista, por mencionar algunos, que en la actualidad estructuran la vida intelectual diaria en todo el mundo (Grafica No 4.) .

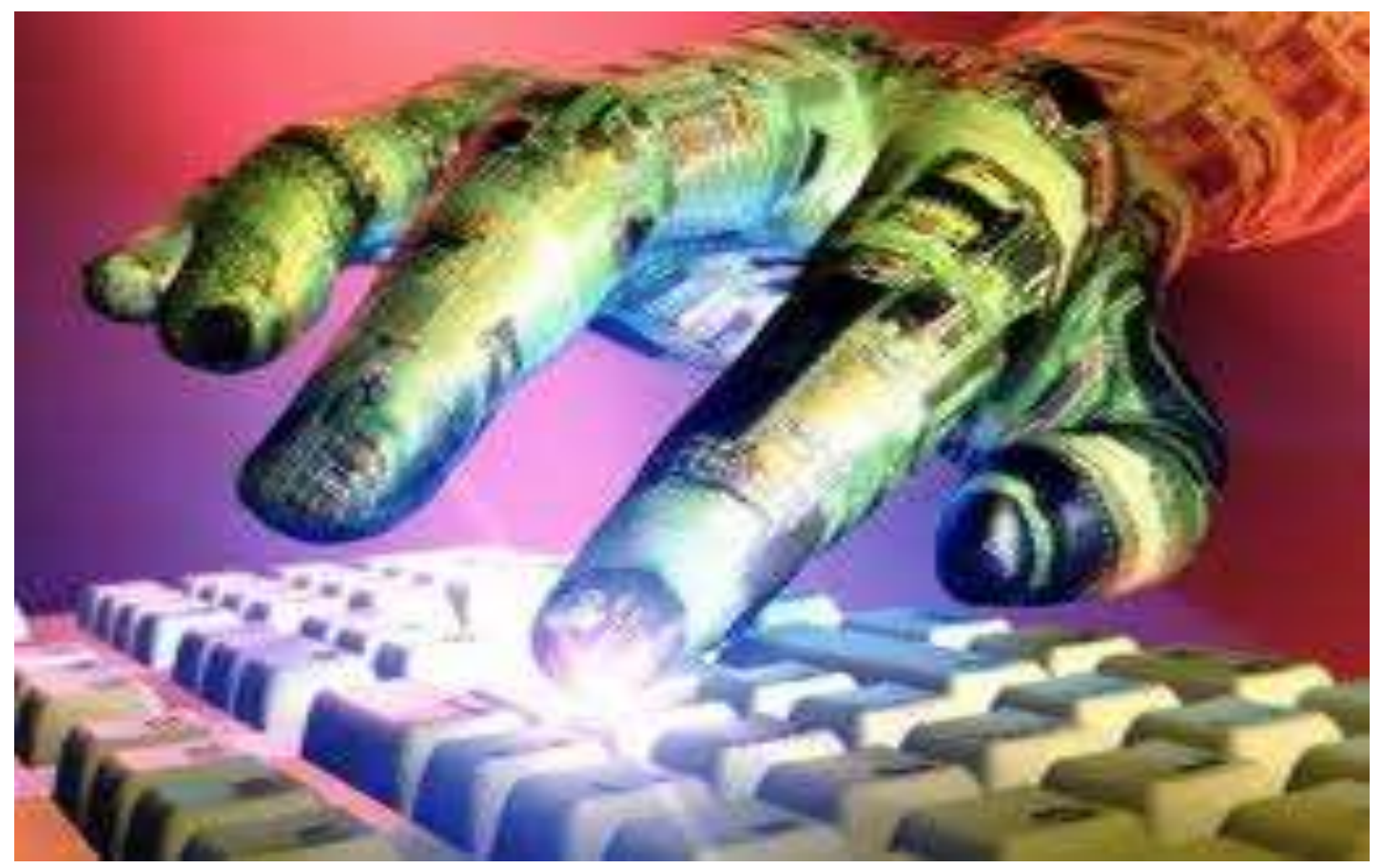

Grafica No 4. Ciudad Digital. Tomado de: pydigital.blogspot.com

Los mercados financieros globales de hoy y las acciones transaccionales del Comercio Electrónico como Amazon.com o de cualquier otra empresa de ese universo de organizaciones comerciales en línea serían imposibles sin una inmensa y muy sofisticada infraestructura Teleinformática. Comunidades Virtuales se han creado por medio de las cuales, se permiten y mantienen conexiones sociales y culturales a través de la operación de aplicaciones 
informáticas como correo electrónico a través de aplicativos como YAHOO, GMAIL, HOTMAIL y otros más, o grupos sociales virtuales soportados en desarrollos teleinformáticos como los de MYSPACE, YOUTUBE, FACEBOOK, entre otros. Sus efectos económicos, sociales y culturales son innegables y se han convertido en expresiones de ideología, mediadores de conciencia e instrumentos de poder muy influyentes que deberán mirarse con un sentido crítico y desde luego ético. Por ejemplo en los casos de Egipto, Libia por mencionar algunas. Como explicar los efectos producidos por las difusiones de la cadena Al Jazeera o las convocatorias realizadas a través de las redes sociales como TWITTER Y FACEBOOK, motivadoras de iniciar y mantener vivas "estas revoluciones" de cambio (Jud, 2011). O como no destacar los efectos causados en diferentes países de este planeta sobre las revelaciones hechas en el portal WIKILEAKS sobre diferentes aspectos políticos y sociales de transcendencia nacional e internacional.

En Colombia es evidente la influencia de gestión de esas comunidades virtuales como una alternativa de organización social en las marchas políticas en todo el país y del planeta Tierra, clamando por la paz para nuestro país o las crisis económicas en Europa o en Estados Unidos. Para el caso Colombiano, recordamos sus inicios en las convocatorias a través de las redes sociales a marchas sociales, realizadas tanto el 4 de febrero, así como la del 6 de marzo de 2008, que fueron encuentros sociales que se convocaron para protestar contra todo tipo de terrorismo, ya sea, el provocado por los grupos de guerrilla paramilitares, o crímenes causados por algunos miembros del estado o de la delincuencia común en Colombia. Como pudo apreciarse en términos generales, el éxito de las marchas fue rotundo no solo por el respeto en que transcurrieron, sino por la gran cantidad de seres humanos que se movilizaron, personas con creencias diferentes, puntos de vista disimiles pero con un acuerdo tácito y explicito común, nuestro país requiere la paz, si queremos armonía en la convivencia y progreso para todos los habitantes de Colombia (Grafica 5.) .

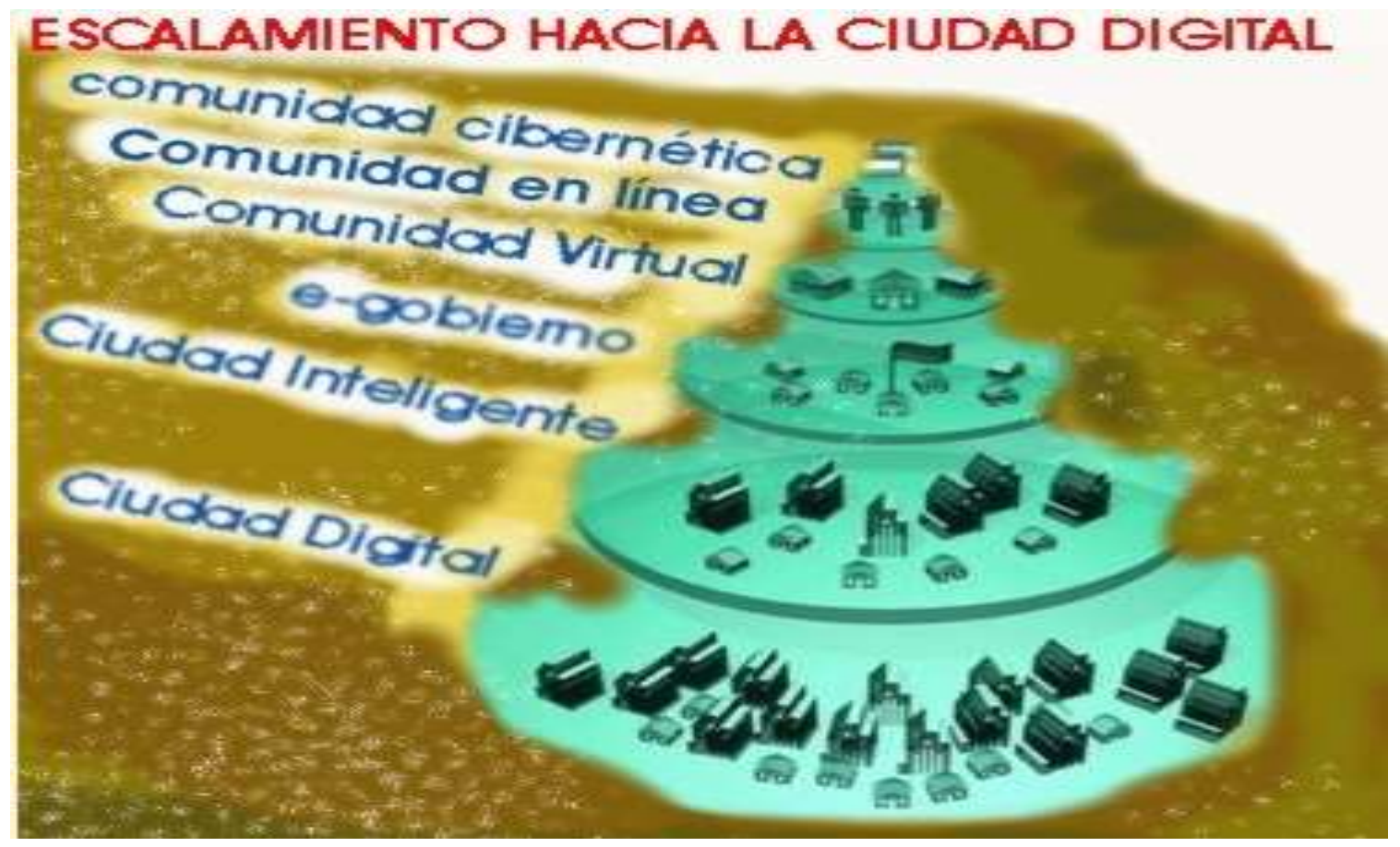

Grafica 5. Ciudad Digital Inteligente. Tomado de: marjoriequelopana.blogspot.com 


\section{CONCLUSIONES}

De todo lo anterior podemos concluir y por tanto reiterar que en la construcción de las Ciudades Digitales está presente una malla creciente de conexiones directas a los sistemas mecánicos, eléctricos, electrónicos e informáticos de los edificios residenciales o comerciales, a los aparatos domésticos, a la maquinaria de producción, a las plantas de procesamiento, a los sistemas de transporte, a las redes eléctricas y a otras redes de suministro de energía, de suministro de agua, evacuación y de eliminación de residuos, a sistemas que proporcionan seguridad vital y sistemas de gestión para casi cualquier actividad humana imaginable.

Por tanto, la esencia de las Ciudades Digitales radica en la combinación cada vez más efectiva de redes de telecomunicación alambricas o inalámbricas (los nervios), la inteligencia integrada de forma ubicua (los cerebros), los sensores e indicadores (los órganos sensoriales) y el software (el conocimiento y la competencia cognitiva) y parece que todo lo anterior es solo el comienzo de lo que nos traerá la multifuncionalidad de los órganos y servicios que pueden ofrecer a la humanidad las TIC en la sostenibilidad de las Ciudades Digitales.

\section{REFERENCIAS BIBLIOGRAFÍCAS:}

- AHCIET (Asociación Hispanoamericana de Centros de Investigación y Empresas de Telecomunicaciones. Manual para el desarrollo de Ciudades Digitales en Iberoamérica.

- BANEGAS, J. (2000): Definición y Concreción del Hipersector de las Tecnologías de la Información en España. Economía de Europa Ciudadano.

- CARAGLIU A., (2009) "Smart cities in Europe". Serie Research Memoranda 0048 (VU University Amsterdam, Faculty of Economics, Business Administration and Econometrics).

- MICHETLL, W. 2003. "Me++: The Cyborg Self and the Networked City".

- VÉLEZ J., 2000 "Arquitectura Virtual: Fronteras", en Construyendo en el espacio digital, Libro de Ponencias del 4to. Congreso de SIGraDi, Río de Janeiro..

\section{INFOGRAFIA:}

- $\quad$ Inf@Vis! La revista digital de InfoVis.net.2002. http://www.infovis.net/printMag.php?num=102\&lang=1

- Perspectivas Microsoft. Revista No 15, 2005. http://www.Microsoft.com

- IX Encuentro Iberoamericano de Ciudades Digitales y cuentan con el apoyo de la Organización de Estados Americanos. 2004. http://www.iberomunicipios.org/home/premio/

- Documentación Ciudades Digitales- http://fcm.org.co/es/

- Ciudades para un Futuro más 2006. http://habitat.aq.upm.es/bpal/onu04/bp1879.html. 
- Un reto para la infraestructura. 2007. http://www.channelplanet.com/index.php

- Jud Andy, 2011. El aliado egipcio está en problemas. http://www.perfil.com/contenidos/2011/01/28/noticia 0019.html.

- Los ecos de Wikileaks. Febrero 22 de 2011. http://www.elespectador.com/noticias/wikileaks/articulo-252139-los-ecos-de-wikileaks.

- La vida en Internet, 2012. http://lavidaeninternet.net/

- Dreamstime, 2012. http://es.dreamstime.com/

- Ciudad Digital, 2012. http://pydigital.blogspot.com/

- Sosteniendo futuro, 2012. http://marioriequelopana.blogspot.com/

- Bogotá - Antigua, 2012: http:// transportationceranayom.qapacity.com

Otras Publicaciones del Autor del presente Artículo:

- Paper sobre "Las Tecnologías de la información y Comunicación-TICS son un caso exitoso en la Educación Media y Básica de Bogotá DC"- organizada por el Centro de Investigación de las Telecomunicaciones - CINTEL y validada por la IEEE (Institute of Electrical and Electronics Engineers) CAl268-2009. En el IV Encuentro de Investigación, Innovación e Ingeniería Tecno bienestar- Bogotá 26 y 27 de agosto de 2009.

- Articulo - "Las TIC como soporte del gobierno electrónico: un caso exitoso en el sector educativo de Bogotá D.C." REVISTA EDUCACIÓN, PEDAGOGÍA Y CIENCIAS II adscrita al ISPInstituto Superior de Pedagogía de la Universidad Autónoma de Colombia - Edición número 2 enero-junio de 2009. ISSN: 2027-3061.

- Ponencia sobre "EI Impacto de las Tecnologías de la Información y la Comunicación-TIC en la operatividad de la Administración Pública de Bogotá D.C. y su relación con el Ciudadano en el Sector de la Educación". Expuesta en el XVI Congreso Internacional sobre Educación Electrónica- Móvil, Virtual y a Distancia-Teleedu- Bogotá D.C. el 18 al 20 de marzo de 2009. Publicado por la Revista Virtual E-mail Educativo: Vol. 1 (2009): OBJETOS VIRTUALES DE APRENDIZAJE. Universidad Nacional de Colombia. Indexación ISSN: 0123 - 4897

- Articulo - "iLas tecnologías inalámbricas RFID y la privacidad humana!" Revista ClepsidraFacultad de Ingeniería de la Universidad Autónoma de Colombia. Edición No 7- Julio -Diciembre de 2008- Indexación la referencia ISSN 1900-1355

- Articulo- "El Impacto de las Tecnologías de la Información y la Comunicación-TICS en la operatividad de la Administración Pública de Bogotá D.C. y su relación con el Ciudadano en el Sector de la Educación" Revista AVANCES - CENTRO DE INVESTIGACIONES de la Universidad Libre . Año 5, Edición No 8, I Semestre de 2008 - Indexación referencia ISSN: 1794-4953.

- Articulo- "La Ingeniería Electrónica en la creación de Redes de Telecomunicaciones". Revista ELIPSE, Edición No 17 de agosto de 2006. Publicada por la Asociación de Ingenieros de la Empresa de Telecomunicaciones de Bogotá - ASIET. Difusión nacional e internacional - Indexación referencia ISSN 1692-0651.

- http://www.latindex.unam.mx/buscador/resBus.html?opcion=1\&exacta=\&palabra=elipse

- Articulo-"La Regulación Colombiana promueve el uso de los Sistemas Inalámbricos". Revista RCT ON LINE, Edición No 11 de julio 4 de 2004. Publicada por el Centro de Investigación de las Telecomunicaciones - CINTEL. Difusión nacional e internacional - Indexación la referencia ISSN 16921585. 
- Articulo-“Las Tecnologías Electrónicas al Servicio de la Humanidad”- Boletín Científico Universitario ENTERESE- Universidad Manuela Beltrán - Edición No 16 agosto de 2004-Indexación la referencia ISSN 0122-7017.

- Articulo-“Las Tecnologías de la Información y la Comunicación TICS, Alcaldías y Desarrollo Social" -Revista ACIEM- Asociación Colombiana de Ingenieros Electrónicos, Mecánicos y Afines-No 717-Diciembre de 2003- Difusión nacional e internacional- Indexación la referencia ISSN 0121-9715.

- Ponencia sobre “La Electrónica en el Área de la Medicina"- VII Jornadas de Electrónica y Electromedicina- organizada por ACIEM- Asociación Colombiana de Ingenieros Electrónicos, Mecánicos y Afines Noviembre de 1993

- Articulo-“La Tecnología Electrónica en la Policía Nacional”- Revista de la Escuela General Santander- No 39 - Marzo de 1988-Difusión nacional e internacional- Indexación la referencia ISSN0120-582XI

- Elaboración de otras publicaciones:

- Coautor del Libro Compendio titulado Gestión Investigativa Ingeniería de Sistemas- Universidad Libre-2009ISBN: 978-958-8534-07-7

- Autor del Libro o Modulo Didáctico Titulado Guía Didáctica y Pedagógica para el curso académico de Instrumentación y Mediciones, Material Pedagógico. Universidad Nacional Abierta y a Distancia-UNAD, 2007. ISBN: 978-958-44-8158-0 